\title{
Bronchoscopic Lung Volume Reduction Using Tissue Engineering Principles
}

\author{
Edward P. Ingenito, Robert L. Berger, A. Cortney Henderson, John J. Reilly, Larry Tsai, and Andrew Hoffman
}

Division of Pulmonary and Critical Care Medicine and Thoracic Surgery, Brigham and Women's Hospital, Boston, Massachusetts; Tufts University School of Veterinary Medicine, North Grafton, Massachusetts; and Bistech, Inc., Woburn, Massachusetts

\begin{abstract}
Bronchoscopic lung volume reduction (BLVR), a minimally invasive procedure based on tissue engineering principles, was performed in six sheep with papain-induced experimental emphysema (EMPH). Physiologic measurements, at baseline, after generation of EMPH, and at 3 and 9 weeks after BLVR, included lung volumes, diffusing capacity $\left(D \mathrm{~L}_{\mathrm{CO}}\right)$, pressure-volume relationships for the lung and chest wall , pleural pressures generated during active respiratory muscle contraction, lung resistance and dynamic elastance. The animal model displayed hyperinflation (change in total lung capacity $+8 \%$; change in residual volume $+66 \%)$, reduced $D_{L_{c o}}(-21 \%)$, and elevated airway resistance $(+76 \%)$ that resembled advanced human EMPH. BLVR was well tolerated without complications, and it reduced lung volumes (change in total lung capacity $-16 \%$; change in residual volume $-55 \%$ ) in a pattern that resulted in significant improvements in vital capacity (10\%). At autopsy, well-organized, peripheral scars associated with tissue contraction were observed at 33 of the $36(91 \%)$ treated sites. There was no evidence of infection, abscess, or granuloma formation, or allergic reaction. Scar tissue, generated by BLVR, replaced hyperinflated lung, reduced overall lung volume, and improved respiratory function safely and consistently. The BLVR technology employed in this study addresses the limitations identified in our prior attempt at BLVR therapy and appears safe and effective enough to justify a trial in humans.
\end{abstract}

Keywords: bronchoscopic volume reduction; lung volume reduction; emphysema; tissue engineering

Lung volume reduction therapy refers to elimination of emphysematous hyperinflated lung, which allows the remaining pulmonary parenchyma and the respiratory muscles to function more effectively (1). Observational studies, and randomized clinical trials, provide convincing evidence that the procedure improves respiratory function, exercise capacity, and symptoms of dyspnea in selected patients with advanced emphysema (EMPH) to a greater extent than optimal medical therapy (1-7). To date, lung volume reduction in humans has been accomplished exclusively through surgical means (lung volume reduction surgery [LVRS]). Although the physiologic and symptomatic benefits of LVRS have, on average, been quite impressive, substantial morbidity and mortality, associated with major thoracic surgery and general anesthesia in an elderly debilitated population, have limited the clinical utility of the procedure $(8,9)$.

We have demonstrated previously that lung volume reduction can be accomplished bronchoscopically, a procedure that represents a potential alternative to LVRS (10). The initial approach to bronchoscopic lung volume reduction (BLVR)

(Received in original form August 9, 2002; accepted in final form October 8, 2002) Supported by HL 62266-03 and Bistech, Inc.

Correspondence and requests for reprints should be addressed to Edward P. Ingenito, M.D., Ph.D., Pulmonary and Critical Care Division, Brigham and Women's Hospital, 75 Francis St., Boston, MA 02115. E-mail: eingenito@partners.org Am J Respir Crit Care Med Vol 167. pp 771-778, 2003 Originally Published in Press as DOI: 10.1164/rccm.200208-842OC on October 11, 2002 Internet address: www.atsjournals.org was based on the simple concept that collapse of target regions could be initiated using a washout solution to disrupt surfactant function, and be maintained with a biocompatible "tissue sealant" to prevent re-expansion. The concept was tested using a sheep model of EMPH, in which lung zones supplied by 5 to $7 \mathrm{~mm}$ airways were blocked, collapsed, and filled with a fibrin hydrogel sealant. This "mechanical" approach to bronchoscopic volume reduction produced atelectasis with subsequent scarring in $55 \%$ of the treated sites, confirming the feasibility of BLVR. However, the procedure was associated with a $15 \%$ incidence of sterile abscess formation (10). Although physiologic benefits in successfully treated sheep were comparable with those of a 20 to $25 \%$ surgical volume reduction, the high failure rate, and unacceptably high incidence of abscess formation rendered this approach to BLVR unacceptable for clinical use.

Recently completed cell culture and large animal studies suggest that to improve the effectiveness and safety of BLVR, consideration of biologic as well as mechanical responses are necessary (11). Successful BLVR essentially requires a remodeling process whereby damaged, hyperinflated lung is transformed into contracted scar by delivering reagents that alter the biologic environment at specific target sites. Although the ultimate objective of the remodeling process is to produce a mechanical response, this can only happen if an appropriate biologic response is first generated. Thus, we hypothesized that redesigning BLVR to improve its safety and effectiveness would require a method that mimics scar formation similar to what occurs during normal healing.

Such responses in the lung are usually initiated by an injury that causes loss of epithelial integrity, followed by inflammation leading to scar formation (12). The BLVR system has been designed to replicate key aspects of this healing process without producing inflammation. This second generation system uses an enzymatic primer solution to remove epithelial cells from the target region and a modified hydrogel scaffold that contains glycosaminoglycans and a synthetic polyamine to promote fibroblast attachment and collagen synthesis. The system was initially tested in control sheep without EMPH; it was well tolerated, and produced efficient scarring (11). The present study describes the application of this novel tissue engineering approach to BLVR in a sheep model of advanced homogeneous EMPH to confirm its safety, evaluate its ability to reduce lung volume, and improve physiologic parameters.

\section{METHODS}

Experiments were conducted in accordance with a protocol approved by the Tufts University School of Veterinary Medicine Institutional Animal Care and Use Committee. All interventions and physiologic measurements were performed under light anesthesia, whereas mechanical ventilation was administered through an endotracheal tube. Anesthesia was induced with intravenous ketamine (Ketaset; Fort Dodge Pharmaceuticals, Fort Dodge, IA, $7.5 \mathrm{mg} / \mathrm{kg}$ ) and diazepam (Valium, $0.25 \mathrm{mg} / \mathrm{kg}$ ) and maintained with continuous intravenous propofol 
(50 $\mu \mathrm{g} / \mathrm{kg} / \mathrm{min}$; Propoflo, Abbott Labs, Columbus, OH). A 10-mmdiameter endotracheal tube was inserted into the trachea under fiberoptic guidance and attached to a mechanical ventilator. An esophageal balloon was introduced through the nares, and the desired position was verified by observing transpulmonary and transrespiratory system pressures, and cardiac oscillations on transpulmonary pressure profiles. Heart rate and arterial oxygen saturation were continuously monitored using an oximeter and tongue probe.

\section{The Experimental Model}

A model for advanced homogeneous EMPH was developed in six female sheep, weighing $67 \pm 22 \mathrm{~kg}$. Seven animals were initially tested and found free of zoonoses (Chlamydia psittaci, Mycobacterium pseudotuberculosis, Brucella, and Coxiella burnetti). Under light anesthesia and controlled mechanical ventilation, Albuterol (five puffs) was administered through an in-line metered-dose inhaler (MDI) device to prevent bronchospasm and to provide uniform distribution of the papain (Sigma Chemical, St. Louis, MO). Papain (75 U/kg weekly for 4 weeks) was delivered over 15 to -20 minutes using a parallel system of dual nebulizers (Pari LC Plus; Pari Respiratory Equipment, Midlothian, VA) connected to the positive pressure ventilator circuit through a series of one-way valves. Tidal volume was set at $12 \mathrm{ml} / \mathrm{kg}$, rate at 12 breaths/ minute, and $\mathrm{FI}_{\mathrm{O}_{2}}$ at 0.6. One of the six animals developed pulmonary hemorrhage after the second dose of papain and was killed. Out of the remaining six animals, two experienced mild respiratory distress and cough after the first and second papain treatments. These clinical signs resolved after treatment with dexamethasone $(0.1 \mathrm{mg} / \mathrm{kg})$. No other adverse reaction to papain was noted.

\section{The BLVR Procedure}

A fiberoptic endoscope (GIF-n30 pediatric gastroscope; Olympus Corporation, Tokyo, Japan) with $5 \mathrm{~mm}$ outside diameter and $90 \mathrm{~cm}$ working length was introduced through the indwelling endotracheal tube and advanced to target segmental bronchi (three in the left lung, and three in the right lung). Ten milliliters of Bistech Primer Reagent $(0.25 \%$ trypsin in buffer phosphate-buffered saline; Bistech Inc., Woburn, MA) was injected through the instrument channel of the bronchoscope and left in place for 2 minutes. Suction was applied to remove as much residual solution as possible. Ten milliliters of Bistech Washout Solution (Roswell Park Memorial Institute-1640 culture media, pH 7.4, containing $10 \%$ heat inactivated fetal bovine serum) was delivered and left in place for 2 minutes to neutralize residual primer. Suction was again applied. Ten milliliters of fibrin hydrogel suspension (3\% fibrin containing $0.1 \%$ chondroitin- 6 -sulfate and $0.1 \%$ poly-L-lysine; Bistech) and $1 \mathrm{ml}$ of thrombin cross-linker $(1,000 \mathrm{U}$ thrombin in $1 \mathrm{ml}$ of phosphatebuffered saline containing $5 \mathrm{mM}$ calcium chloride) were delivered simultaneously at this site through a dual lumen catheter system. These relatively small volume injectates were used to help avoid pressure necrosis and sterile abscess formation that had been observed in prior studies using injection volumes of $50 \mathrm{ml}$. This procedure was repeated at each of the six treatment sites. Individual treatments required approximately 5 to 7 minutes to complete, and the entire BLVR protocol took 30 to 45 minutes. After completion of BLVR therapy, anesthesia was discontinued and animals were monitored closely during recovery. Once clinically stable, each animal was returned to its stall.

The following six sites were selected for treatment in each sheep: (1) the dorsal segment of the right cardiac lobe, (2) the medial segment of the right diaphragmatic lobe, (3) the caudal segment of the right diaphragmatic lobe, (4) the cranial segment of the left apical lobe, (5) the lateral segment of the left diaphragmatic lobe, and (6) the caudal segment of the left diaphragmatic lobe. These sites included both dependent and nondependent portions of the lung and were easily identified during bronchoscopy via direct visualization.

\section{Description of Experimental Protocol}

Under anesthesia and controlled mechanical ventilation, detailed respiratory physiology measurements were performed and computerized tomography (CT) scans of the chest were obtained at baseline (BAS), 2 weeks after receiving a full course of papain on development of experimental EMPH but just before BLVR (EMPH), and 3 and 9 weeks after BLVR. Clinical status including activity levels, body weights, and vital signs were monitored during follow-up. On completion of the 9-week post-BLVR studies, the animals were killed and autopsied. Gross and microscopic examinations were performed, and representative photographic documentation was obtained.

\section{Physiologic Measurements}

(1) Lung volume (functional residual capacity [FRC]) was measured by whole body plethysmography during spontaneous breathing (D. Buxco Electronics, Sharon, CT). Intermittent airway occlusions were triggered remotely (outside the box) using a pneumatic shutter (Pneumatic controller valve; Hans Rudolf Inc., Kansas City, MO), allowing for measurements of inspiratory efforts against a closed shutter and determination of intrathoracic gas volume based on Boyle's law (10). (2) Pleural pressure was obtained during inspiratory efforts against a closed shutter at FRC and FRC $+1.5 \mathrm{~L}$, to characterize the pressurevolume $(\mathrm{PV})$ relationship for the chest wall during respiratory muscle contraction. (3) The deflation PV relationship for the lung measured from $30 \mathrm{~cm} \mathrm{H}_{2} \mathrm{O}$ transpulmonary distending pressure was determined by intermittent occlusion. (4) The PV relationship for the chest wall was measured in identical fashion to characterize the compliance characteristics of the relaxed chest wall in the absence of muscle contraction. (5) Airway resistance, tissue resistance, and dynamic elastance measurements were determined to characterize the dynamic properties of the lung using the optimal ventilator waveform approach of Lutchen and coworkers (13) with a computer-controlled large animal ventilator. (6) Single breath diffusing capacity $\left(\mathrm{DL}_{\mathrm{CO}}\right)$ was measured by filling the lung to total lung capacity (TLC) with tracer gas $(10 \%$ helium $/ 0.3 \%$ carbon monoxide $/ 21 \%$ oxygen/balanced nitrogen) via a supersyringe, and analyzing the expirate using a carbon monoxide/helium analyzer. These measurements essentially replicate the standard battery of clinical tests used to assess patients for LVRS.

Chest CT scans were performed at resting end expiratory lung volumes (FRC) and at full lung inflation (TLC).

\section{Analysis of Physiologic Data}

Static PV data for the lungs were fit to the exponential equation of Salazaar and Knowles (14), in which lung volume was expressed as a function of pressure according to the equation:

$$
\mathrm{V}(\mathrm{P})=\mathrm{Vmax}-\mathrm{Ae}^{-\mathrm{kP}}
$$

where $\mathrm{V}$ is volume, $\mathrm{P}$ pressure, $\mathrm{Vmax}$ the volume attained at "infinite" distending pressure, $\mathrm{A}=(\mathrm{Vmax}-\mathrm{Vmin}), \mathrm{Vmin}$ is the volume at zero distending pressure (equivalent to residual volume $[\mathrm{RV}]$ ), and $\mathrm{k}$ is a parameter describing the shape of the exponential relationship between pressure and volume (the shape factor). Results are reported in terms of the parameters Vmax, Vmin, and $\mathrm{k}$. TLC is determined as the volume intercept of the active chest wall PV relationship and the passive lung $\mathrm{PV}$ relationship. The corresponding pressure at this point of intercept is the recoil pressure at TLC.

Dynamic lung function measurements obtained using the optimal ventilator waveform $(\mathrm{OVW})$ technique were interpreted by fitting frequency domain expressions for pressure, volume, and flow data to the constant phase impedance model of Hantos and coworkers (15):

$$
\mathrm{P}(\omega) / \mathrm{V}(\omega)=\mathrm{Raw}+\left\{(\mathrm{G}-\mathrm{H} j) / \omega^{\alpha}\right\}
$$

where $\mathrm{P}$ is pressure, $\mathrm{V}$ is flow, $\omega$ is angular frequency in radians/second, $j$ is the imaginary number $V-1$, Raw is airway resistance, and other terms are as previously defined. Results are summarized in terms of airway resistance, tissue resistance (expressed as the dissipative modulus $\mathrm{G}$ ), and dynamic elastance (expressed as the elastic modulus $\mathrm{H}$ ).

Pleural pressure deflections measured during inspiratory efforts against a closed shutter were assessed at FRC, and at FRC + 1.5 L. The maximum inspiratory effort generated during a 60- to 90-second occlusion was recorded as the maximal inspiratory pleural pressure at the corresponding lung volume. Inspiratory PV data were used to construct an "active chest wall" compliance relationship that was assumed to be linear between TLC and RV.

$\mathrm{DL}_{\mathrm{CO}}$ was determined in intubated, anesthetized sheep using an airtight three-way valve system, a calibrated carbon monoxide and helium meter (Morgan Transfer factor system; Morgan Scientific, Haverhill, MA), source of calibrated tracer gas, and a 3-L syringe. A volume of $\mathrm{DL}_{\mathrm{CO}}$-calibration gas equal to the inspiratory capacity for the sheep was drawn into the syringe, with the sheep lung volume at 
FRC. The calibrated tracer gas was then injected into the sheep and full inflation maintained for 10 seconds. The injected volume of gas was withdrawn, and the expirate injected back through the meter to determine the concentration of expired carbon monoxide and helium. The alveolar volume was calculated from a single breath helium dilution measurement. Using alveolar volume and the breath hold time, $\mathrm{DL}_{\mathrm{CO}}$ can then be calculated as: $\mathrm{DL}_{\mathrm{CO}}=\left[\mathrm{VA} /\left(\mathrm{Pb}-\mathrm{PH}_{2} \mathrm{O}\right)\right] \times 60 /$ breath hold time $\times \ln \left(\mathrm{Fi}_{\mathrm{Aco}} / \mathrm{Ff}_{\mathrm{Aco}}\right)$. $\mathrm{V}_{\mathrm{A}}$ is the alveolar volume, $\mathrm{Ff}_{\mathrm{Aco}}$ is the measured fractional concentration of carbon monoxide at the end of the breath hold, $\mathrm{Fi}_{\mathrm{Aco}}$, the initial alveolar concentration of carbon monoxide is equal to the injected concentration of carbon monoxide $\times$ injected syringe volume/TLC.

\section{Pathologic Anatomy}

After completion of 9-week post-BLVR physiologic measurements, the animals were killed with intravenous pentobarbital $(100 \mathrm{mg} / \mathrm{kg})$ and autopsied. The lungs were removed, fully inflated, and the pulmonary circulation was then flushed with $2.5 \mathrm{~L}$ of normal saline at $20 \mathrm{~cm} \mathrm{H}_{2} \mathrm{O}$ pressure. Sites of BLVR were readily detected by visual inspection and manual palpation, and were photographed. The lungs were sliced serially into 2 to $2.5 \mathrm{~cm}$ axial sections and further inspected for signs of scar formation or other gross changes. Samples from all BLVR treatment sites and representative samples from untreated sites were fixed in $15 \%$ buffered formalin and stained with hemotoxylin and eosin.

\section{Statistical Analysis}

Comparisons between physiologic parameters at different time points were performed by analysis of variance for repeated measures. Differences between specific groups were identified using Duncan's post hoc analysis test. Comparisons among nonparametric outcome variables were performed by $\chi^{2}$ analysis. Statistical significance was defined as a $\mathrm{p}$ value of less than 0.05 .

\section{RESULTS}

BAS and EMPH lung and chest wall physiology are compared in Figure 1. Static lung and chest wall mechanics are presented in the form of a Campbell diagram analogous to the approach previously used by Fessler and Permutt (16) to describe physiologic responses to LVRS. This single figure summarizes the interaction between the chest wall and lung that is responsible for the hyperinflation, gas-trapping, and loss of recoil characteristic of EMPH. The figure also illustrates how volume reduction reduces gas trapping and restores recoil pressures through resizing of the lung within the thorax.

The animal model of papain-induced EMPH displayed hyperinflation (TLC increased from $3.36 \pm 0.36$ to $3.63 \pm 0.42 \mathrm{~L}, \mathrm{p}=$ 0.09 and FRC from $1.72 \pm 0.23$ to $2.04 \pm 0.27, \mathrm{p}=0.003)$, gas trapping ( $\mathrm{RV}$ increased from $0.86 \pm 41$ to $1.43 \pm 0.48, \mathrm{p}=$ $0.009)$, and a decrease in elastic recoil pressure $(15.7 \pm 3.1$ to $8.1 \pm 2.7, \mathrm{p}=0.003)$. In addition, airway resistance (before $=$ $0.45 \pm 0.31$; after $=0.79 \pm 0.127, \mathrm{p}=0.035)$ and dynamic elastance (before $=16.9 \pm 4.2$; after $=19.6 \pm 3.4, \mathrm{p}=0.153$ ) increased. These values closely resemble changes observed in humans with advanced EMPH (17). Papain treatment caused a substantial decrease in $\mathrm{DL}_{\mathrm{CO}}$ by $20.3 \%$ (from $47.9 \pm 13.7$ to $36.1 \pm 12.2 \mathrm{ml} / \mathrm{min} / \mathrm{mm} \mathrm{Hg}, \mathrm{p}=0.019$ ) indicating the presence of tissue destruction and the loss of alveolar surface for gas exchange.

The BLVR procedure was well tolerated. None of the animals developed hypoxemia, hemoptysis, bronchospasm, or respiratory distress either during or after treatment. Arterial oxygen saturation values measured before starting the procedure, and 10 minutes after completion of the procedure on the same ventilator settings $\left(\mathrm{FI}_{\mathrm{O}_{2}}=0.6\right.$, tidal volume $=12 \mathrm{ml} / \mathrm{kg}$, breathing rate $=$ $12 / \mathrm{min}$, and positive end expiratory pressure $=0$ ) were nearly identical $\left(\mathrm{Sa}_{\mathrm{O}_{2}}\right.$ : before $=96.8 \pm 0.8 \%$; after $\left.=95.3 \pm 2.4 \%\right)$. Supplemental oxygen therapy or reintubation was not required in any of the sheep. All animals were active, eating, and behaving

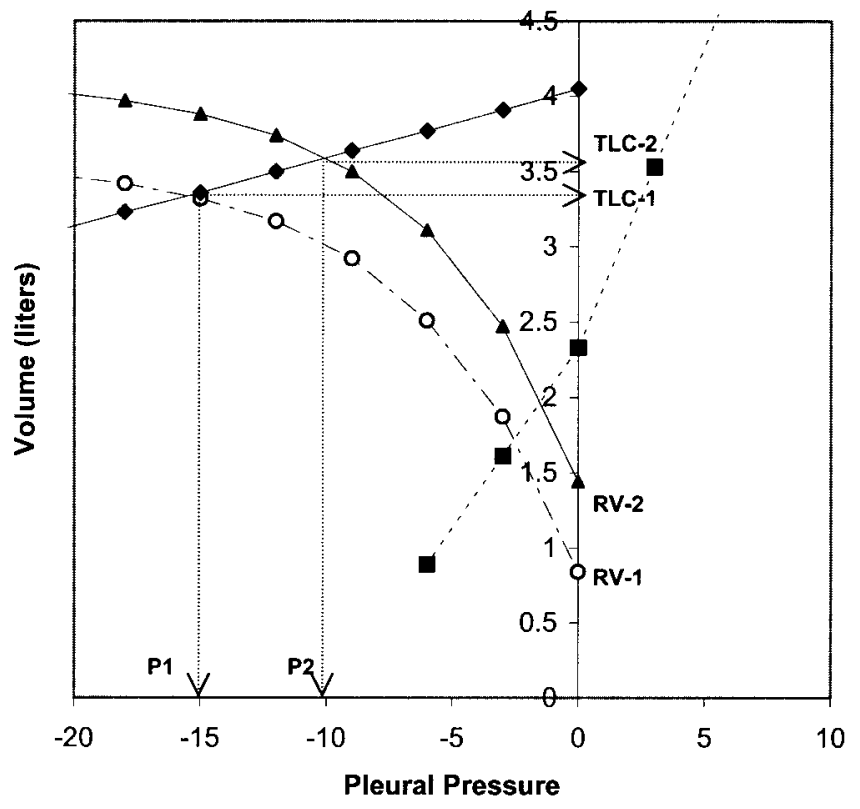

$-\rightarrow-\mathrm{CL}(\mathrm{BAS}) \multimap \mathrm{CW} \longrightarrow \mathrm{Cl}$ - - CWpass $\longrightarrow-\mathrm{CL}(\mathrm{EMPH})$

Figure 1. The Campbell diagram summarizes the relationship between lung and chest wall mechanics in normal sheep (BAS), and in homogeneous $E M P H$. With EMPH, the $C L$ curve shifts upward. QSPV curves for the lung, CWpass, and CWact are shown. TLC and RV increased in response to papain treatment indicating hyperinflation and gas trapping. Recoil pressure decreased (from $P 1$ to $P 2$ ). $C L=$ lung compliance; CWpass = passive chest wall; $C W a c t=$ active chest wall; $T L C=$ total lung capacity; $R V=$ residual volume; $T L C-1=$ baseline; $T L C-2=$ after papain; $R V-1=$ baseline; $R V-2$ = after papain.

normally within 24 hours of undergoing BLVR. Body weights remained stable pre- to 9 weeks postprocedure. One of the six sheep experienced a fever immediately after BLVR but defervesced without specific therapy over 36 hours. No other adverse clinical events were observed.

The 3-week post-BLVR physiologic measurements revealed significant reductions in TLC (from $3.63 \pm 0.42$ to $3.01 \pm 0.32 \mathrm{~L}$, $\mathrm{p}=0.02$ ), FRC (from $2.04 \pm 0.27$ to $1.66 \pm 0.37 \mathrm{~L}, \mathrm{p}=0.05$ ), and RV (from $1.43 \pm 48$ to $0.63 \pm 0.17 \mathrm{~L}, \mathrm{p}=0.002$ ). On average, each injection produced a $2.8 \%$ reduction in overall lung volume, resulting in a $17 \%$ reduction from treatment at six sites. This is approximately equivalent to a $50 \%$ reduction in the total volume of lung initially subtended by these airways. Active and passive chest wall PV relationships did not significantly change in response to BLVR therapy. The improvements in lung function observed 3 weeks after treatment were still present and were similar in magnitude at the 9-week follow-up. The Campbell diagram (Figure 2) summarizing these results shows that the volume reduction, and resizing of lung to chest wall produced by this procedure not only decreased trapped gas (RV-2 to RV-4) and lung volumes (TLC-2 to TLC-4), but also increased recoil pressures (P2 to $\mathrm{P} 4)$.

A summary of the effects of BLVR on lung volumes throughout the study period is presented in Figure 3. Animals with papain-induced EMPH exhibited increases in RV and TLC, a decrease in vital capacity, and an increase in RV/TLC ratio from a BAS of 0.25 to 0.41 . BLVR was followed by decreases in RV and TLC, an increase in vital capacity, and a decrease in RV/ 


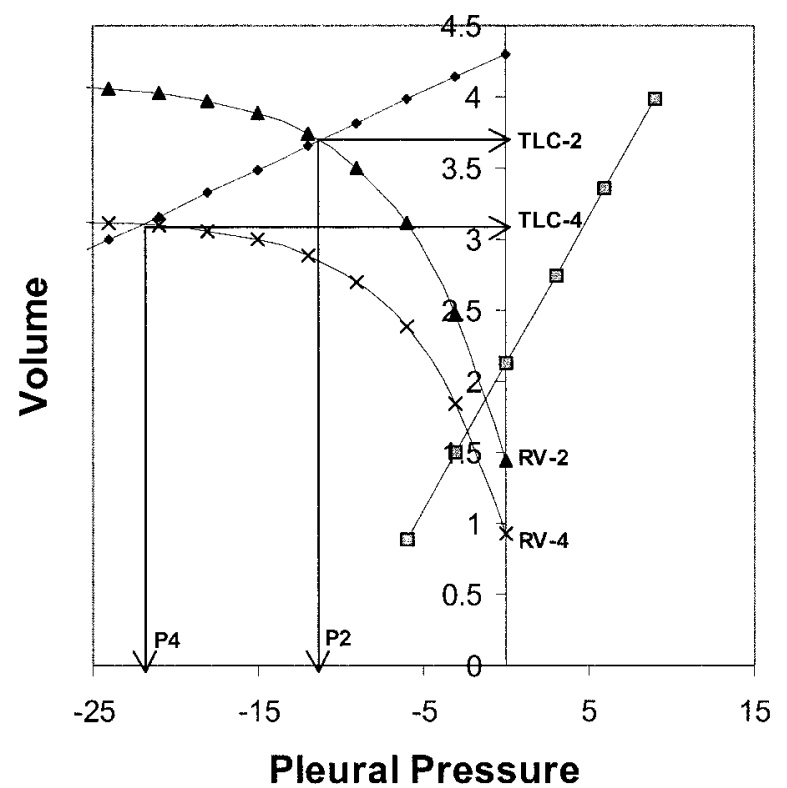

- Cwact - Cwpass $\triangle \mathrm{CL}(\mathrm{EMPH}) \times \mathrm{CL}(\mathrm{BVR}-2)$

Figure 2. The Campbell diagram summarizes the response to BLVR in sheep with homogeneous EMPH 9 weeks after treatment. TLC-4 and RV-4 are significantly reduced (TLC-2 and $R V-2$ ). Recoil pressure (P4) increased significantly from pretreatment $E M P H$ values (P2). CWact and CWpass mechanics were unaffected by BLVR. Results 3 weeks after BLVR are not shown but are similar to those recorded 9 week after follow-up (BVR-2). CL = lung compliance; CWact = active chest wall; CWpass = passive chest wall; $R V=$ residual volume; $R V-2=$ after papain; $R V-4=$ 9 weeks after BLVR; $T L C=$ total lung capacity; $T L C-1=$ baseline; $T L C-2=$ after papain; $T L C-4=9$ weeks after BLVR.

TLC ratio to 0.21 at 3 weeks. At 9 weeks RV/TLC ratio remained decreased at 0.26 .

Airway resistance increased after papain administration but returned to pre-EMPH BAS values by 9 weeks post-BLVR. Tissue resistance and dynamic elastance were unaffected by treatment. $\mathrm{DL}_{\mathrm{CO}}$ declined significantly after papain exposure but showed a trend back toward BAS at 9 weeks. This value did not differ appreciably from that observed at BAS before papain

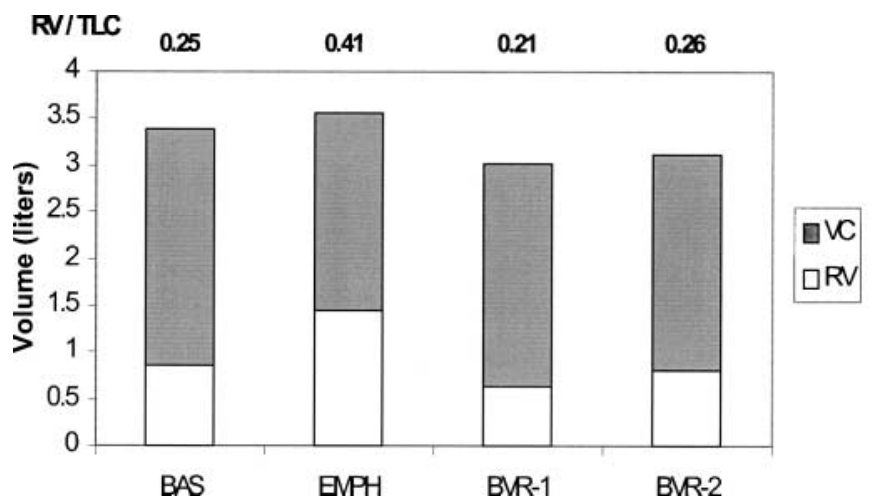

Figure 3. Physiologic measurements obtained from homogeneous EMPH study animals are shown in a stacked bar graph format. $R V=$ residual volume, $T L C=$ total lung capacity, and $V C=$ vital capacity. RV/TLC ratio increased after EMPH was established but fell after BLVR.

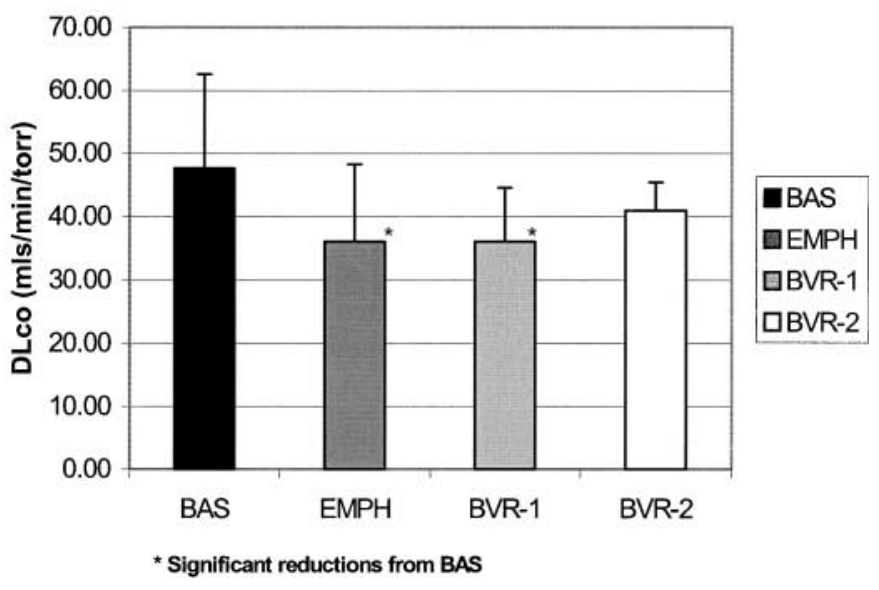

Figure 4. $D \mathrm{~L}_{\mathrm{CO}}$ diffusing capacity at baseline $(B A S)$, after development of emphysema (EMPH), 3 weeks after BLVR (BLVR-1), and 9 weeks after $\operatorname{BLVR}(B L V R-2)$. At $B L V R-2$, the $\mathrm{D}_{\mathrm{CO}}$ value and was not appreciably different from $B A S$.

$(47.9 \pm 13.7$ at BAS versus $41.0 \pm 4.5 \mathrm{ml} / \mathrm{min} / \mathrm{mm} \mathrm{Hg}$ at 9 weeks after BLVR; $p=0.31$ ) (Figure 4).

Serial CT scans provided visual documentation of the changes produced by BLVR treatment (Figure 5). At BAS before BLVR (Figure 5A) the target area selected for treatment showed no abnormal densities. Immediately after BLVR, collapse and loss of volume are demonstrated at the treatment site (Figure 5B). At 9 weeks, the treatment area had undergone volume contraction, with development of a localized linear scar (Figure 5C).

Autopsy, performed 9 weeks after BLVR, showed grossly visible, well-demarcated, zones of whitish-appearing scar tissue on the pleural surfaces of the lung at 33 of the 36 targeted sites in the six animals (Figure 6). These locations corresponded to sites of fibrin hydrogel injections and to the linear densities observed on CT scan.

Microscopic examination of the lungs revealed that papain exposure had produced diffuse tissue destruction with heterogeneity in air space size. The pattern of destruction resulting from papain nebulization was most consistent with panlobular destruction. At sites of BLVR treatment, extensive collagen deposition, with collections of fibroblasts and mononuclear cells, consistent with organized scar tissue was observed. There was no suggestion of pneumonitis, granuloma formation, residual foreign body material, or evidence of tissue necrosis or abscess formation (Figures 7 and 8).

\section{DISCUSSION}

The present study represents the application of a novel strategy for treating pulmonary EMPH by promoting formation of scar tissue to replace diseased hyperinflated lung parenchyma. Because the scar tissue occupies a much smaller volume than the associated air-filled and hyperexpanded lung from which it derives, the process reduces overall lung volume. Although no new functioning lung tissue is created during BLVR, the resizing of the lung ameliorates a mismatch between the hyperinflated lung and the nonyielding rigid chest cage. This anatomic disparity is a fundamental disturbance that governs the pathophysiology of EMPH, and the resizing that follows volume reduction therapy significantly improves lung function. In contrast to more traditional LVRS, BLVR achieves this resizing without the major trauma and potential morbidity of surgery.

The sheep model of advanced homogenous EMPH used in 

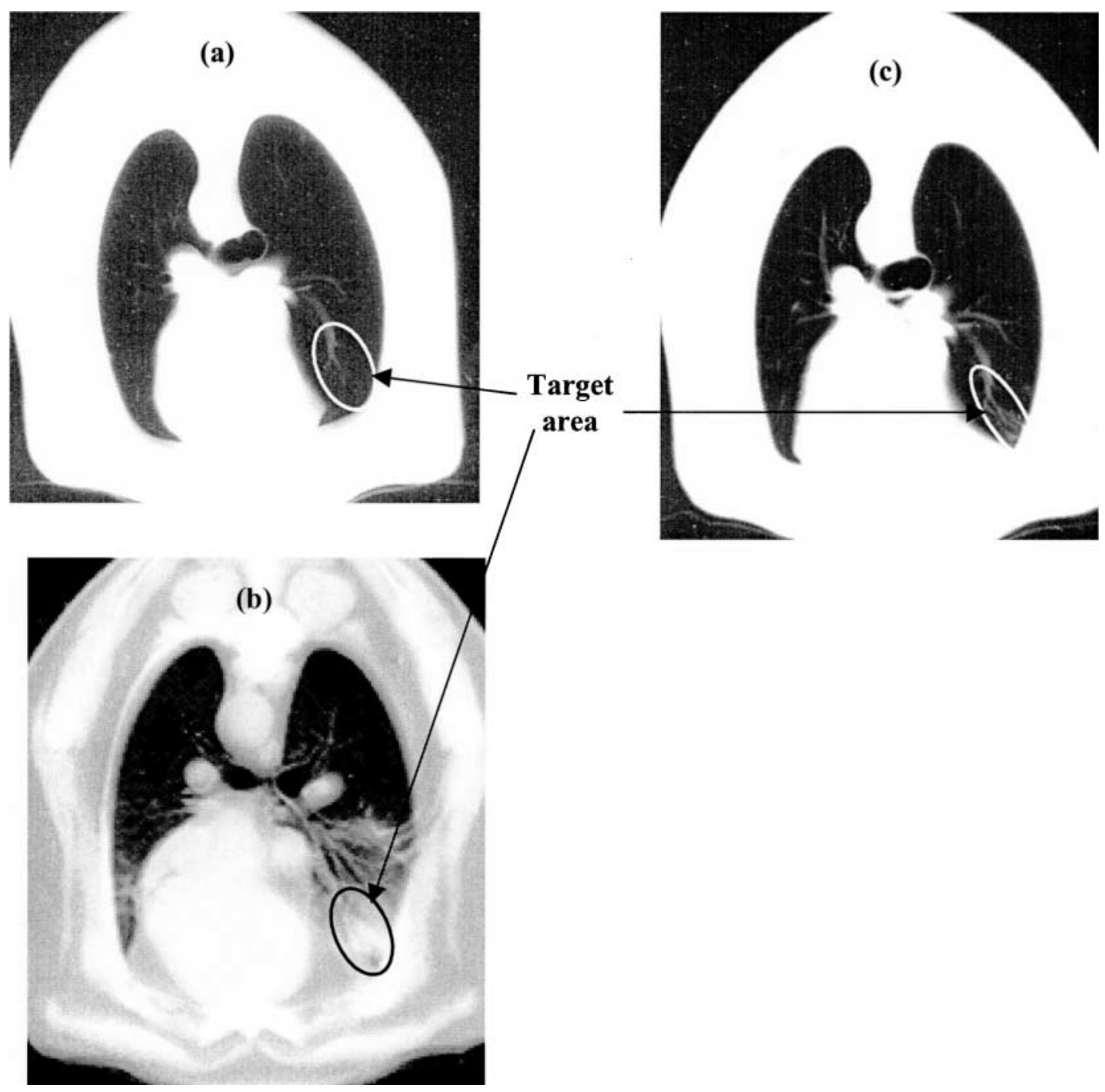

Figure 5. Serial CT images of the chest from sheep 9912 showing a treatment site $(A)$ after papain administration but before BLVR, $(B)$ immediately after BLVR, and 9 weeks later (C). The changes in (B) suggest consolidation that is replaced by a linear density consistent with scar tissue 9 weeks later (C).

the present study displayed a more severe form of disease than the model employed in previous BVLR experiments. In this earlier experimental model of mild-to-moderate EMPH, papain was administered during spontaneous breathing (10). In the present study, nebulized papain was delivered after bronchodilator therapy and during positive pressure mechanical ventilation using relatively large tidal volumes $(12 \mathrm{ml} / \mathrm{kg})$. This modification in technique produced more severe tissue damage and correspondingly greater physiologic disturbances without detectable pleural or parenchymal scarring or persistent inflammation observed at 9-week follow-up. One animal did develop respiratory distress and pulmonary hemorrhage after two doses of papain and was killed. The remaining six tolerated the papain treatments with only mild side effects.

The physiologic disturbances after papain exposure were of moderate severity and were evident 2 weeks after completion of treatments. RV, an indicator of trapped gas, rose by $66 \%$ but TLC increased by only $8 \%$. The disproportionate increment in RV relative to TLC resulted in an impressive decline both in vital capacity (21\%) and elastic recoil pressure $(48 \%)$. $\mathrm{DL}_{\mathrm{CO}}$ also fell by $21 \%$. The tissue destruction found at autopsy explains the profound physiologic changes and marked loss of surface area for gas exchange.

Because this experimental model displayed homogeneous EMPH, target sites of severe damage were not evident. Physio- logic responses to volume reduction were therefore not dependent on the location of treatment sites but rather the total number of treatment sites. The sites treated with BLVR included both dependent and nondependent areas to permit evaluation of the BLVR system's ability to safely treat areas located throughout all regions of the lung. Results presented here confirm that target areas can be readily identified without imaging guidance and that both dependent and nondependent regions can be safely treated without reagent migration or spill-over into adjacent nontarget areas of lung.

BLVR was performed in a fashion nearly identical to conventional bronchoalveolar lavage and was well tolerated by all sheep. No major adverse systemic responses were observed during or after completion of the procedures. The animals recovered quickly and did not manifest clinically detectable respiratory distress. They were standing, walking, and eating within an hour of removal of the endotracheal tube and discontinuation of anesthesia (propofol), and resumed normal activity within hours of BLVR therapy.

CT scan images showed local consolidation and collapse in the target territory immediately after BLVR. These early radiographic changes evolved into linear densities on CT image after 9 weeks (Figure 5) and corresponded to the location of peripheral scars observed at necropsy. There was no evidence of pleural effusion, pleural scarring, or lung abscesses at any of the treatment sites. 


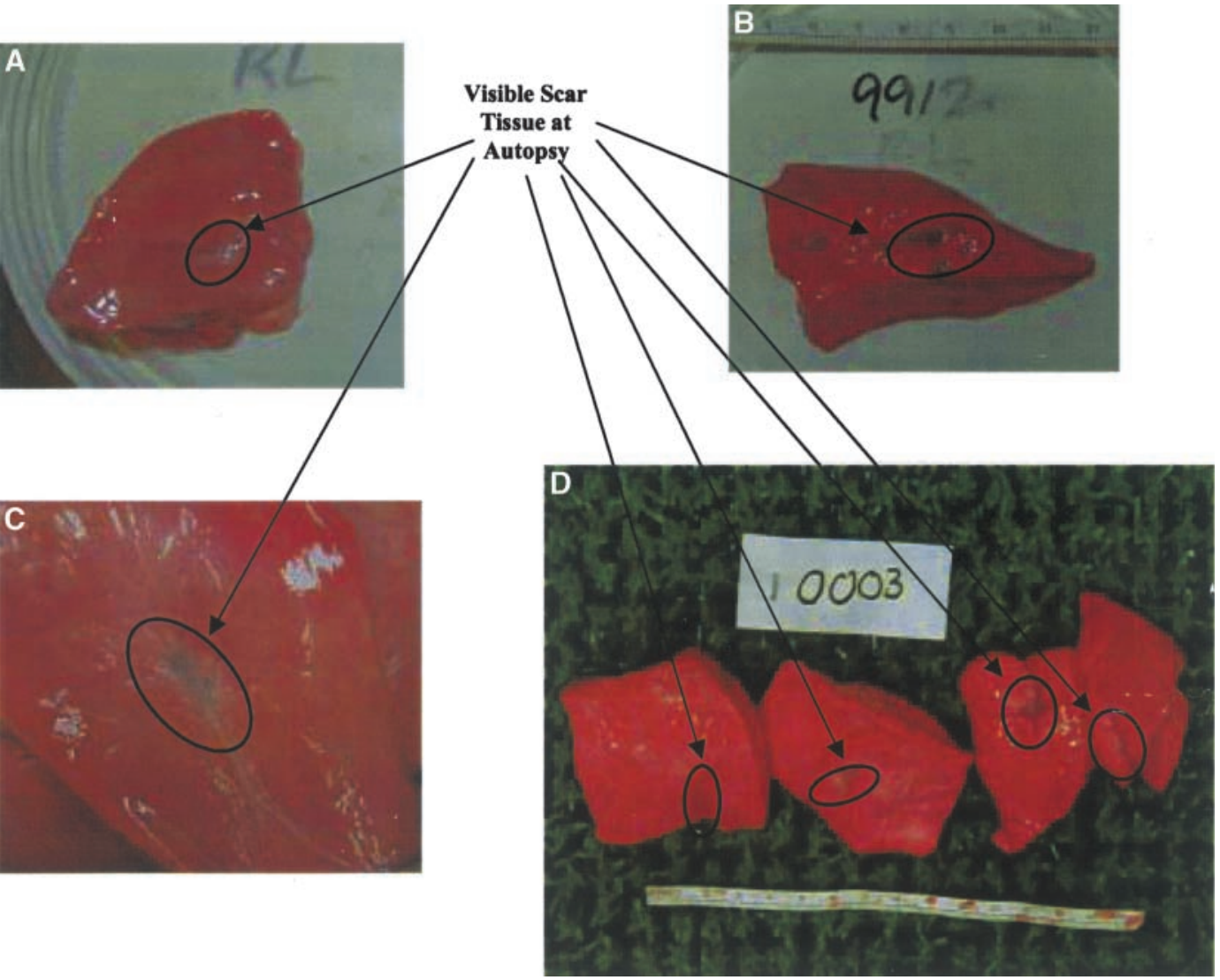

Figure 6. Cut surfaces of the lungs show scar tissue at BLVR treatment sites in four different animals are shown in $(A)-(D)$.

BLVR was followed by impressive reductions in both TLC $(17 \%$ at 3 weeks and $16 \%$ at 9 weeks, $p=0.02)$ and $\mathrm{RV}(56 \%$ at 3 weeks, $p=0.002 ; 41 \%$ at 9 weeks, $p=0.01)$. Analysis of static lung volumes by the Campbell diagram indicates that the improvements in vital capacity and recoil pressure observed were largely due to a decrease in trapped gas volume (RV). At 3 weeks post-BLVR, part of the reduction in gas volume may have been due to consolidation in the target territory from the injection of reagents. However, these areas of parenchymal consolidation radiographically evolved into linear densities, and the organized, contracted scar tissue found at necropsy 6 weeks later, suggests that lung volume was getting smaller. Anatomic findings combined with improvements in physiologic indices provide strong evidence for a cause and effect relationship between BLVR-induced scar formation and volume reduction.

Our initial attempt at BLVR had focused on a mechanical approach for generating volume reduction through occlusion of segmental bronchi using relatively large aliquots $(50 \mathrm{ml})$ of fibrin hydrogel. The results were somewhat disappointing because only $55 \%(11 / 20)$ of the targeted areas demonstrated sustained atelectasis and scar. In addition, there was a $15 \%(3 / 20)$ incidence of sterile abscess formation. We postulated that the failure to produce sustained collapse at all target sites might have been due to potent endogenous plasmin activity at the lung epithelial surface leading to rapid degradation of fibrin gel at some injection sites. We further postulated that sterile abscesses were probably the result of pressure necrosis due to filling of lobules with relatively large volumes of hydrogel injectate that on crosslinking generate local hydrostatic pressures that exceed capillary perfusion pressure. Concerns regarding effectiveness as well as safety of the original BLVR approach, led to the development of a new generation BLVR based on biologic modulation rather than mechanical obstruction (11). As summarized here, results were substantially better using this modified approach. Effectiveness rose from 55 to $91 \%$ (33/36 versus 11/20 sites with scar formation, $\mathrm{p}<0.001$ by $\chi^{2}$ ), and safety improved as demonstrated by the drop in sterile abscess formation from 15 to $0 \%(0 / 36$ sites versus $3 / 20$ sites with sterile abscess formation, $p=0.025$ by $\chi^{2}$ ).

Simple mechanical approaches to volume reduction have been tested by other investigators and have met with problems. At least two groups have placed occlusive materials and devices within the proximal airways to generate volume reduction, but to our knowledge no appreciable lasting physiologic benefits have been obtained $(18,19)$. In one instance, the procedure was associated with serious complications, including pneumothorax, pneumonia, and hemorrhage (19). Inability to collapse target areas using devices deployed within the proximal bronchi has been attributed to extensive collateral ventilation within the periphery of the EMPH lung $(20,21)$. In patients with advanced disease, resistance to airflow through collateral channels may be equivalent to or less than that through anatomic airways, making volume reduction using a strategy based on proximal obstruction difficult, if not impossible. In those areas where collapse can be achieved, retained secretions can predispose to postobstructive infections.

This new, nonsurgical BLVR system based on principles of tissue engineering involves the safe, controlled removal of bronchial epithelial lining cells and deployment of a fibrin hydrogel into the airways of hyperinflated emphysematous areas of lung to provide a scaffold for fibroblast chemotaxis and attachment, leading to the evolution of localized scar tissue formation. The $91 \%$ success rate of achieving scar formation in the targeted territory and the associated improvement in pulmonary function 

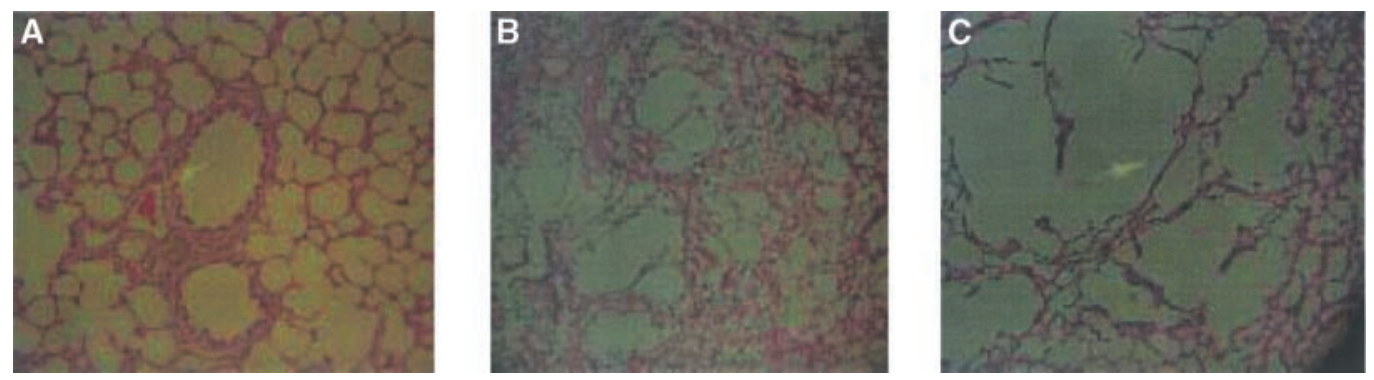

Figure 7. Hemotoxylin and eosin stained lung tissue samples are shown from a control animal at $\times 100$ magnification $(A)$ and from an animal with induced $\mathrm{EMPH}$ at $\times 40(B)$ and at $\times 100$ (C) magnification.

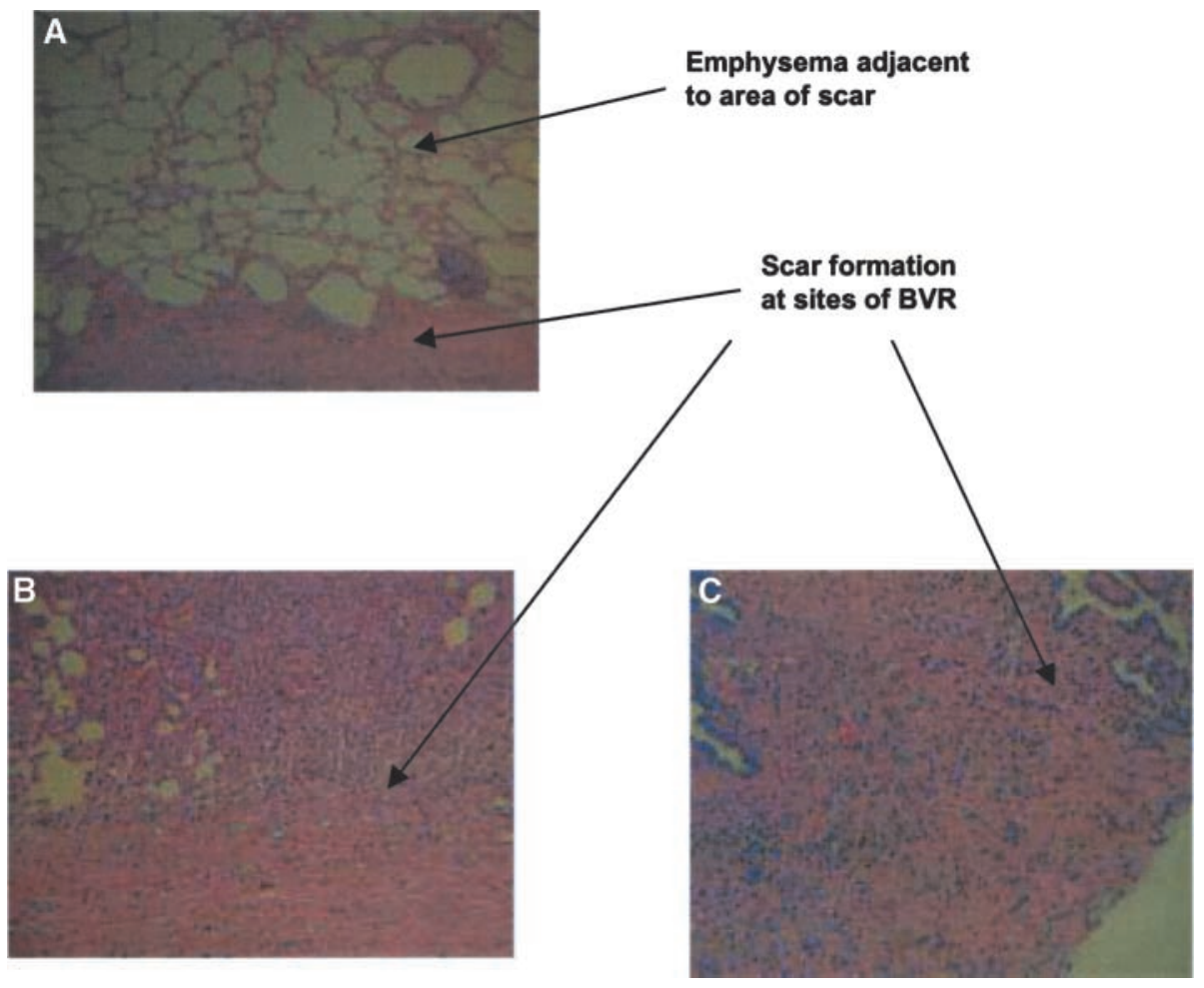

Figure 8. Hemotoxylin and eosin stained tissue at a treated site shows well-organized scar tissue with adjacent nontreated emphysematous lung under $\times 100$ magnification 9 weeks after $B L V R(A)$, well organized scar alone under $\times 100(B)$ and under $\times 200$ magnification $(C)$.

suggest that the scar shrinks the hyperinflated lung and reduces overall lung volume. The absence of sterile abscess formation indicates that this second-generation BLVR procedure addresses the safety concerns associated with the initial approach.

Although these data support the effectiveness and safety of this new procedure as applied to sheep with EMPH, differences in physiology between the animal model and human disease could influence anticipated clinical responses. The sheep studied here demonstrated substantial tissue destruction and hyperinflation. However, gas exchange was affected to a lesser degree. Resting room air saturations in sheep after papain exposure before BLVR ranged from 93 to $97 \%$. By contrast, the majority of patients considered for volume reduction therapy require supplemental oxygen to maintain arterial saturation levels greater than $90 \%$. Therefore, this sheep model does not accurately reproduce the hypoxemia observed in humans with advanced EMPH who are candidates for volume reduction. It is therefore possible that BLVR could pose a greater risk of procedure-related hypoxemia in patients than was observed in sheep. However, several factors mitigate against this supposition. Only the most damaged and dysfunctional regions of lung are targeted for BLVR treatment. The ability to target only these regions for volume reduction minimizes the likelihood of producing new areas of $\dot{\mathrm{V}} / \dot{\mathrm{Q}}$ imbalance during the procedure, and therefore reduces the tendency for acute hypoxemia. It can even be argued that larger disturbances in gas exchange might be anticipated in the sheep that have less BAS lung damage and are therefore more prone to acute hypoxemia as a result of development of new zones of altered $\dot{V} / \dot{Q}$ during BLVR. In our studies, little acute desaturation was, in fact, observed, and all animals recovered rapidly from BLVR without evidence of respiratory insufficiency. Thus, it can be reasonably argued that this modified approach to BLVR that uses small volumes of injectate and targets only the most dysfunctional areas of lung is unlikely to cause significant alterations in arterial oxygenation in patients, even when abnormalities in BAS gas exchange exist.

The absence of BAS hypoxemia in this model also precludes evaluation of any beneficial effects of BLVR on oxygenation. However, we do not consider this to be a significant limitation because volume reduction therapy has not been shown to produce consistent clinical benefits with respect to oxygenation (22), and thus such benefits are not necessarily expected from BLVR. Improvement in respiratory function after volume reduction therapy occurs independent of effects on oxygenation.

Although differences in patterns of collateral ventilation do, in fact, exist between humans and ruminants in general, studies 
have shown that collateral ventilation in the sheep and human lung is quite similar (23). Furthermore, the success of this procedure is not jeopardized by the presence of extensive collateral ventilation. Hydrogel polymerization occurs within seconds of injection, filling distal airspaces to serve as a scaffold for cell growth and proliferation. Once the scaffold is established, scarring and contraction occur over a period of weeks as illustrated by the serial CT scans presented here, and thus successful BLVR does not require immediate mechanical collapse.

In summary, the present study demonstrates that tissue engineering-based BLVR approaches designed to reduce lung volume by promoting localized fibroblast proliferation, collagen synthesis, and scar formation achieved success in $91 \%$ of the targeted territory without causing acute hypoxemia, infection, or sterile abscess formation. The procedure technically is similar to bronchoalveolar lavage performed at the level of the segmental to subsegmental airways. Each treatment causes, on average, a 2 to $3 \%$ reduction in overall lung volume. Scarring remained localized and did not spread beyond the treatment zones. There was no evidence of tissue dysplasia or anaplasia, allergic reaction, or systemic organ injury. The magnitude of physiologic improvement observed in this experimental model would be expected to benefit humans with EMPH to an extent similar to that produced by LVRS. Thus, second generation BLVR, which relies on cell modulation, provides an effective, safer alternative to LVRS than our previous BLVR technique based on the principle of mechanical obstruction.

\section{References}

1. Cooper JD, Patterson GS, Sundaresan RS, Trulock EP, Yusen RD, Pohl MS, Lefrak SS. Results of 150 consecutive bilateral lung volume reduction procedures in patients with severe emphysema. J Thorac Cardiovasc Surg 1996;112:1319-1329.

2. Benditt JO, Lewis S, Wood DE, Klima L, Albert RK. Lung volume reduction surgery improves maximal $\mathrm{O}_{2}$ consumption, maximal minute ventilation, $\mathrm{O}_{2}$ pulse, and dead space to tidal volume ratio during leg ergometry. Am J Respir Crit Care Med 1997;156:561-566.

3. Moy ML, Ingenito EP, Mentzer SJ, Evans RB, Reilly JJ. Health-related quality of life improves following pulmonary rehabilitation and lung volume reduction surgery. Chest 1999;115:383-389.

4. Criner GJ, Cordova SC, Furukawa S, Kuzma AM, Travaline JM, Leyenson V, O'Brien GM. Prospective randomized trial comparing bilateral lung volume reduction surgery to pulmonary rehabilitation in severe chronic obstructive pulmonary disease. Am J Respir Crit Care Med 1999;160:2021-2027.

5. Geddes D, Davies M, Koyama H, Hansell D, Pastorino U, Pepper J, Agent P, Cullinan P, MacNeill SJ, Goldstraw P. Effects of lung volume reduction in patients with severe emphysema. $N$ Engl $J$ Med 2000;343:239-245.

6. Goodnight-White S, Jones WJ, Baaklini J, Soltero E, Smithwick P, Sharafkhaneh A, Officer T, Reinoso MA, Manian P, Miller C, Rodarte JR. Prospective randomized controlled trial comparing bilateral lung volume reduction surgery (LVRS) to medical therapy alone in patients with emphysema. Chest 2000;118:102s.

7. Pompeo E, Marino M, Nofroni J, Matteucci G, Mineo TC. Reduction pneumoplasty versus respiratory rehabilitation alone in severe emphysema: a randomized study. Ann Thorac Surg 2000;70:948-954.

8. Swanson SJ, Mentzer SJ, DeCamp MM, Bueno R, Richards WG, Ingenito EP, Reilly JJ, Sugarbaker DJ. No-cut thoracoscopic lung placation: a new technique for lung volume reduction surgery. J Am Coll Surg 1997; 85:25-32.

9. Utz JP, Hubmayr RD, Deschamps C. Lung volume reduction surgery for emphysema: out on a limb without a NETT. Mayo Clin Proc 1998; 73:552-566.

10. Ingenito EP, Reilly JJ, Mentzer SJ, Swanson SJ, Vin R, Keuhn H, Berger RL, Hoffman A. Bronchoscopic volume reduction: a safe and effective alternative to surgical therapy for emphysema. Am J Respir Crit Care Med 2001;164:295-301.

11. Ingenito EP, Tsai L, Gilmartin G, Reilly JJ, Henderson C, Keuhn H, Hoffman A. Bronchoscopic volume reduction using biopolymers which target fibroblasts, macrophages, and epithelial cell responses. Am J Respir Crit Care Med 2001;163:A957.

12. Rennard, SI. Inflammation and repair processes in chronic obstructive pulmonary disease. Am J Respir Crit Care Med. 1999;160:s12-s16.

13. Lutchen KR, Yang K, Kaczka DW, Suki B. Optimal ventilation waveforms for estimating low-frequency respiratory impedance. J Appl Physiol 1993;75:478-488.

14. Salazaar E, Knowles JH. An analysis of pressure-volume characteristics of the lung. J Appl Physiol 1964;19:97-104.

15. Hantos Z, Adamicza A, Govaerts E, Daroczy B. Mechanical impedances of lungs and chest wall in the cat. J Appl Physiol 1992;73:427-433.

16. Fessler HE, Permutt S. Lung volume reduction surgery and airflow limitation. Am J Respir Crit Care Med 1998;157:715-722.

17. Kaczka DW, Ingenito EP, Lutchen KR. A technique to determine inspiratory impedance during mechanical ventilation: implications for flow limited patients. Ann Biomed Eng 1999;27:1-16.

18. Wantanabe Y. LVRS with WBA. World Bronchology Conference, Boston, MA; June 2-6, 2002.

19. Snell GI, Smith JA, Silvers AJ, Thompson KR, Rosenfeldt FL, Holsworth L, Williams TJ. Bronchoscopic volume reduction: a pilot study. American College of Chest Physicians Meeting, October 21-25, 2001.

20. Morrell NW, Wignall BK, Biggs T, Seed WA. Collateral ventilation and gas exchange in emphysema. Am J Respir Crit Care Med 1994;150: 635-641.

21. Hogg JG, Macklem PT, Thurlbeck WM. The resistance of collateral channels in excised human lungs. J Clin Invest 1969;48:412-431.

22. Albert RK, Benditt JO, Hildebrandt J, Wood E, Hlastala MP. Lung volume reduction surgery has variable effects on blood gases in patients with emphysema. Am J Respir Crit Care Med 1998;158:71-76.

23. Kuriyama T, Wagner WW. Collateral ventilation may protect against high-altitude pulmonary hypertension. J Appl Physiol Respir Environ Exerc Physiol 1981;51:1251-1256. 